\title{
TWO CONVEX COUNTEREXAMPLES: A DISCONTINUOUS ENVELOPE FUNCTION AND A NONDIFFERENTIABLE NEAREST-POINT MAPPING
}

\section{J. B. KRUSKAL}

Introduction. This paper gives counterexamples for two unrelated conjectures which pertain to convexity theory.

Suppose $f$ is any function defined on a closed convex set $C$ in finitedimensional Euclidean space. Its envelope function env $f$ is defined as the pointwise supremum of all linear functions which $f$ dominates everywhere. It is elementary and well known that env $f$ is convex, and hence continuous in the relative interior of $C$. It is similarly known that env $f$ is lower-semicontinuous on the boundary of $C$. Witsenhausen [14] uses and studies envelope functions. He suggested the following orally:

Conjecture 1. If $f$ is continuous everywhere on $C$, then env $f$ is also continuous everywhere on $C$.

I provide a very simple counterexample in 3-space which shows that this is not true. Incidentally, though the proof is not on paper, I feel fairly certain that Witsenhausen's conjecture is correct in 2-space.

If $C$ is a closed convex set in finite-dimensional Euclidean space, then for any point $x$ in the space there is a unique point $P(x)$ in $C$ which is nearest to $x$. The mapping $P$ is called the projection onto $C$. (Many interesting results about this mapping are given in Phelps [9], [10].) It is easy to prove and well known that $P$ is a contraction mapping, hence continuous. It is also easy to see that $P$ need not have much in the way of differentiability properties, for if $C$ is a polyhedron in 2-space, then it is easy to find points and directions at which $P$ does not even have a two-sided directional derivative. The existence in general of a one-sided directional derivative, however, is not so easy to decide. This question does not seem to be raised in such natural places as Eggleston [4] and Valentine [11].

Conjecture 2. For all points and directions, $P$ has a one-sided directional derivative.

My mildly complicated counterexample is a set $C$ (in 3-space), which is the convex hull of a countable infinity of points. I suspect that the conjecture is correct in 2 -space. In $n$-space it would be interesting to know whether the set of points and directions at which the conjecture holds is dense in $E_{n} \times S_{n-1}$. If not, the convex set would have to be extremely jagged!

Received bv the editors February 19, 1969. 
Counterexample to Conjecture 1. Envelope functions are used by Hans Witsenhausen [14] and by other authors under other names (for example, in an important paper J. J. Moreau [7] calls it the " $\Gamma$-regularized of $f$ "). Suppose $f$ is any function defined on some closed convex set $c$ in $E_{n}$. We say that a (nonhomogeneous) linear function

$$
u(x)=a+\sum_{1}^{n} b_{i} x_{i}
$$

is dominated by $f$ if $u(x) \leqq f(x)$ for all $x$ in $C$. Let $L(f)$ be the set of all linear functions dominated by $f$, and define the envelope function env $f$ by

$$
\operatorname{env} f(x)=\sup _{u \operatorname{in} L(f)}\{u(x)\} .
$$

The authors mentioned point out the following simple facts: Because env $f$ is the supremum of convex (even linear) functions, it is convex, hence continuous on the (relative) interior of $C$. As the supremum of continuous functions, env $f$ must be lower-semicontinuous everywhere, which is pertinent on the (relative) boundary of $C$.

In his paper, Witsenhausen faces the situation where the original function $f$ is known to be continuous. For his control theory application, he wished to prove continuity of env $f$ on the boundary of $C$. He was able to prove (Proposition 31 on p. 20 of [14]) only two special cases: if $C$ is a convex polyhedron, that is, the convex hull of a finite set, then env $f$ is continuous on $C$; or if $x$ is an exposed boundary point of $C$, that is, if there is a hyperplane which meets $C$ only in $x$, then env $f$ is continuous at $x$. However, he conjectured (oral communication) that continuity of $f$ always guaranteed the continuity of env $f$.

The key to the counterexample is the structure of the "faces" of the convex set. If $C$ is closed convex, and $H$ is any supporting hyperplane (that is, a hyperplane which meets $C$ but does not separate it), then the intersection of $C$ and $H$ is called a face of $C$. This immediately generalizes the faces of a polyhedron. An $e d g e$ means a 1-dimensional face, and a vertex means a 0 -dimensional face. Note that a vertex means exactly the same thing as an exposed point.

In the counterexample, $C$ has vertices (exposed points) whose limiting point is in the relative interior of an edge. This is the central fact which makes the example work. Figure 1 displays $C$.

In 3 -space, denote the coordinates by $a, b$, and $c$. The function $f$ is extremely simple: $f(x)=-c^{2}$. The region $C$ is the convex hull of a circle in the coordinate $(a, b)$-plane (call this the "equator") and a 
line segment of length 2 parallel to the $c$-axis, having its midpoint on the equator (call this midpoint the "special point"). Except at the special point, every point of the equator is an exposed point.

The proof that env $f$ is discontinuous at the special point is quite simple. Witsenhausen shows without difficulty (Proposition 31, proof of Case 2), that if $f$ is continuous at an exposed point, then env $f=f$ at that point. This shows that env $f$ is 0 on the equator, excepting the special point. It also shows that env $f$ is -1 at the ends of the line segment. Since env $f$ is convex, it must never exceed -1 along the line segment. Thus env $f$ is discontinuous at the special point.

As a matter of fact, env $f(a, b, c)=-|c|$ except along the line segment, where it is -1 . Thus it is discontinuous all along this segment, except at the ends. The reader may easily verify this.

Counterexample to Conjecture 2. Moreau [7] defines the projection function onto a closed convex set $C$ and calls it proj or $\operatorname{proj}_{C}$. With Nashed (1968), we call it $P$. For any $x$ in $E_{n}, P(x)$ is defined to be the unique point of $C$ which is nearest to $x$. This definition directly generalizes projections on to a linear subspace. Moreau notes the simple fact that if $x_{1}$ and $x_{2}$ are any two points in the space, then distance $\left(P\left(x_{1}\right), P\left(x_{2}\right)\right) \leqq$ distance $\left(x_{1}, x_{2}\right)$, that is, $P$ is a contraction mapping. As an elementary consequence, $P$ is continuous.

In line with standard definitions, the directional derivative of $P$ at $x$ in the direction $u$ (the vector $u$ is assumed to have length 1 ) is given by

$$
\lim _{\epsilon \rightarrow 0} \frac{P(x+\epsilon u)-P(x)}{\|(x+\epsilon u)-x\|}=\lim _{\epsilon \rightarrow 0} \frac{P(x+\epsilon u)-P(x)}{\epsilon} .
$$

If $\epsilon$ is restricted to approach 0 from the positive side, then this becomes the one-sided directional derivative.

By considering convex polygons in the plane, it is easy to see that the two-sided directional derivative of $P$ may often fail to exist. However, in such simple examples the one-sided directional derivative does exist. Must it always? This question arose quite naturally during the examination of the differentiability properties of leastsquares monotone regression (for material on monotone regression, see Barton and Mallows [1], Bartholomew [2] and [3], Kruskal [5], Miles [6], and van Eeden [12] and [13]). The earliest known reference to monotone regression is Ayer, Brunk, Ewing, Ried, and Silver$\operatorname{man}[0]$.

The idea behind our example is this. We shall have points $x_{i}$ which approach a limit $x_{\infty}$ from some fixed direction. The projections 
$p_{i}=P\left(x_{i}\right)$ will of course approach $p_{\infty}=P\left(x_{\infty}\right)$. However, we will arrange matters so that for $e v e n$ values of $i$ the vector $p_{i}-p_{\infty}$ will point almost in one direction (converging to that direction as $i \rightarrow \infty$ ), while for $o d d$ values of $i$ the vector $p_{i}-p_{\infty}$ will converge to a different direction. This is enough to assure nonexistence of the one-sided derivative. Figure 1 displays the example, with considerable liberties of scale to gain visual clarity.

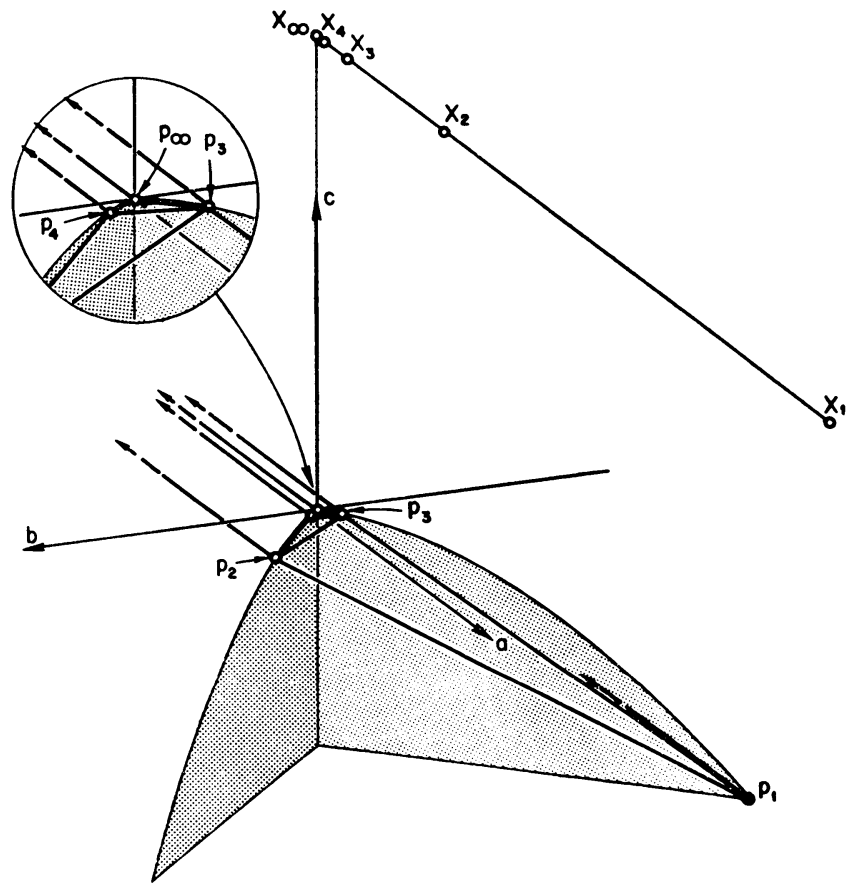

FIgURE 1. Note that scale has been freely altered to improve visual clarity.

In 3-space denote the coordinates by $a, b, c$. To visualize the counterexample, first imagine the two curves described in parametric form by $a(s)=s, b(s)=s, c(s)=-s^{2}$ and $a(s)=s, b(s)=-s, c(s)=-s^{2}$. For some suitable number $\alpha<1$ (later we will use $\alpha=0.01$ and $\alpha$ $=0.25)$, imagine the points for which $s=\alpha^{i}$ for $i=1,2,3, \cdots$ Along the first curve, we use the points for which $i$ is even, along the second curve the points for which $i$ is odd. Both of these sequences converge to the origin, which we add to the collection. Finally, we add the point $a=-R, b=0, c=0$ (with $R$ large and positive) to the collection. The set $C$ is the convex hull of all these points.

The point $x$ where we prove the derivative not to exist is $a=0$, 
$b=0, c=1$. The projection of $x$ on $C$ is the origin. The direction in which the one-sided derivative does not exist is $a=1, b=0, c=0$. To see this, imagine a point $y$ approaching $x$ from this direction. The projection of $y$ on $C$ lies on the two curves alternately (as well as lying between them most of the time) for $y$ arbitrarily close to $x$. (This is what requires calculation.) Thus the ratio used to define the derivative gets close to both the line $b=a$ and close to the line $b=-a$ infinitely of ten as $y$ approaches $x$, and thus does not converge.

Define $C$ to be the closed convex hull of the following countable collection of points: $q=[-R, 0,0], p_{i}=\left[\alpha^{i},(-1)^{i} \alpha^{i},-\left(\alpha^{i}\right)^{2}\right]$ for $i \geqq 0$, and $p_{\infty}=[0,0,0]$, where $R$ is either $\infty$ or some large positive number, and $\alpha>0$ is some suitable small number (for example, $\alpha=0.01)$. Then $P([0,0,1])=[0,0,0]$, and the one-sided derivative of $P$ at $[0,0,1]$ in the direction $[1,0,0]$ does not exist.

In particular, for $\lambda>1$, let $x_{i}=\left[\lambda \alpha^{i}, 0,1\right]$ for $i \geqq 0$, and $x_{\infty}=[0,0,1]$. Then $x_{i}$ approaches $x_{\infty}$ from the positive $[1,0,0]$ direction.

We shall establish by direct calculation that given suitable parameter values (for example, $\lambda=10$ and $\alpha=0.01$ ), $P\left(x_{i}\right)=p_{i}$ and $P\left(x_{\infty}\right)$ $=p_{\infty}$. Then trivially,

$$
\begin{aligned}
& \frac{P\left(x_{i}\right)-P\left(x_{\infty}\right)}{\left\|x_{i}-x_{\infty}\right\|} \\
& \quad=\frac{p_{i}-p_{\infty}}{\lambda \alpha^{i}} \\
& \quad=(1 / \lambda)\left[1,(-1)^{i},-\alpha^{i}\right] \rightarrow \begin{cases}(1 / \lambda)(1,1,0) & \text { if } i \text { even and } i \rightarrow \infty, \\
(1 / \lambda)(1,-1,0) & \text { if } i \text { odd and } i \rightarrow \infty .\end{cases}
\end{aligned}
$$

This shows that the limit which defines the one-sided derivative does not exist.

It only remains to verify that the $p$ 's are the projections of the $x$ 's for suitable parameter values. It is obvious that $P\left(x_{\infty}\right)=p_{\infty}$. To show that $P\left(x_{i}\right)=p_{i}$, it is enough to show that all the points $p_{j}$ and $q$ (which define $C$ ) lie behind the hyperplane through $p_{i}$ which is normal to $p_{i}-x_{i}$. Thus it suffices to show that

$$
\begin{aligned}
\left(p_{i}-x_{i}\right) \cdot\left(p_{i}-p_{j}\right) & \leqq 0 \\
\left(p_{i}-x_{i}\right) \cdot\left(p_{i}-q\right) & \leqq 0
\end{aligned}
$$

for all $i$ and $j$. The latter inequality is obvious. By direct calculation, (1) can be written as follows, where $w=\alpha^{j-i}$ :

$$
\alpha^{2 i}\left\{(1-\lambda)(1-w)+\left(1-(-1)^{i+j_{w}}\right)+\left(1+\alpha^{2 i}\right)\left(1-w^{2}\right)\right\} \leqq 0 .
$$


Dividing by $\alpha^{2 i}$, and noting that $-(-1)^{i+i} w \leqq+w$, we see that it suffices to show that

$$
-\lambda(1-w)+\left(3-w^{2}\right)+\alpha^{2 i}\left(1-w^{2}\right) \leqq 0 .
$$

For $j=i$, (1) is trivial, so we assume $j \neq i$ in (3). For $j>i$, $w<1$, so (3) becomes

$$
\lambda \geqq \frac{3-w^{2}}{1-w}+\alpha^{2 i}(1+w)=\frac{2}{1-w}+(1+w)\left(1+\alpha^{2 i}\right) .
$$

As $w=\alpha^{i-i} \leqq \alpha$ in this case, and $\alpha^{2 i} \leqq \alpha^{2}$, this holds whenever

$$
\lambda \geqq \lambda_{1} \equiv \frac{2}{1-\alpha}+(1+\alpha)\left(1+\alpha^{2}\right) .
$$

For $j<i, w>1$, so (3) becomes

$$
\lambda \leqq \frac{w^{2}-3}{w-1}+\alpha^{2 i}(w+1)=-\frac{2}{w-1}+(1+w)\left(1+\alpha^{2 i}\right) .
$$

As $w=\alpha^{j-i} \geqq 1 / \alpha$ in this case, and $\alpha^{2 i} \geqq 0$, this holds whenever

$$
\lambda \leqq \lambda_{2} \equiv-\frac{2 \alpha}{1-\alpha}+\frac{1+\alpha}{\alpha} .
$$

Brief numerical calculation now reveals that for values of $\alpha \leqq \alpha_{0}$ $\approx 0.264, \lambda_{1} \leqq \lambda_{2}$ so that solutions exist. Among these solutions are $\alpha=0.25, \lambda=4$, and $\alpha=0.01, \lambda=10$.

\section{REFERENCES}

0. Miriam Ayer, H. D. Brunk, G. M. Ewing, W. T. Reid, and Edward Silverman, An empirical distribution function for sampling with incomplete information, Ann. Math. Statist. 26 (1955), 641-647.

1. D. E. Barton and C. L. Mallows, The randomization bases of the amalgamation of weighted means, J. Roy. Statist. Soc. Ser. B 23 (1961), 423-433.

2. D. J. Bartholomew, A test of homogeneity for ordered alternatives, Biometrika 46 (1959), 36-48.

3. - A test of homogeneity of means under restricted alternatives (with discussion), J. Roy. Statist. Soc. Ser. B 23 (1961), 239-281.

4. H. G. Eggleston, Convexity, Cambridge Univ. Press, Cambridge, England, 1963.

5. J. Kruskal, Multidimensional scaling by optimizing goodness-of-fit to a nonmetric hypothesis, Psychometrika 29 (1964), 1-28.

6. R. E. Miles, The complete amalgamation into blocks, by weighted means, of a finite set of real numbers, Biometrika 46 (1959), 317-327.

7. Jean Jacques Moreau, "Convexity and duality" in Functional analysis and optimization, E. R. Caianiello (editor), Academic Press, New York, 1966.

8. M. Z. Nashed, A decomposition relative to convex sets, Proc. Amer. Math. Soc. 
19 (1968), 782-786.

9. R. R. Phelps, Convex sets and nearest points, Proc. Amer. Math. Soc. 8, (1957), 790-797.

10. - Convex sets and nearest points. II, Proc. Amer. Math. Soc. 9 (1958), 867-873.

11. F. A. Valentine, Convex sets, McGraw-Hill, New York, 1964.

12. C. van Eeden, Maximum likelihood estimation of partially or completely ordered parameters. I, Nederl. Akad. Wetensch. Proc. Ser. A 60=Indag. Math. 19 (1957), 128-136.

13. - Note on two methods for estimating ordered parameters of probability distribution, Nederl. Akad. Wetensch. Proc. Ser. A 60=Indag. Math. 19 (1957), 506512.

14. H. Witsenhausen, A minimax control problem for sampled linear systems, IEEE Trans. Automatic Control AC-13 (1968), 5-21.

Bell Telephone laboratories, Incorporated, Murray Hill, New Jersey 